\title{
DEQUANTIZING COMPRESSED SENSING WITH NON-GAUSSIAN CONSTRAINTS
}

\author{
L. Jacques ${ }^{1,2}$, D. K. Hammond ${ }^{1}$, M. J. Fadili ${ }^{3}$ \\ ${ }^{1}$ Institute of Electrical Engineering, Ecole Polytechnique Fédérale de Lausanne (EPFL), CH-1015 Lausanne, Switzerland \\ ${ }^{2}$ Communications and Remote Sensing Laboratory, Université catholique de Louvain (UCL), B-1348 Louvain-la-Neuve, Belgium \\ ${ }^{3}$ GREYC CNRS-ENSICAEN-Université de Caen, 14050 Caen France
}

\begin{abstract}
In this paper, following the Compressed Sensing paradigm, we study the problem of recovering sparse or compressible signals from uniformly quantized measurements. We present a new class of convex optimization programs, or decoders, coined Basis Pursuit DeQuantizer of moment $p\left(\mathrm{BPDQ}_{p}\right)$, that model the quantization distortion more faithfully than the commonly used Basis Pursuit DeNoise (BPDN) program. Our decoders proceed by minimizing the sparsity of the signal to be reconstructed while enforcing a data fidelity term of bounded $\ell_{p}$-norm, for $2<p \leqslant \infty$.

We show that in oversampled situations the performance of the $\mathrm{BPDQ}_{p}$ decoders are significantly better than that of BPDN, with reconstruction error due to quantization divided by $\sqrt{p+1}$. This reduction relies on a modified Restricted Isometry Property of the sensing matrix expressed in the $\ell_{p^{-}}$ norm $\left(\mathrm{RIP}_{p}\right)$; a property satisfied by Gaussian random matrices with high probability. We conclude with numerical experiments comparing $\mathrm{BPDQ}_{p}$ and $\mathrm{BPDN}$ for signal and image reconstruction problems.
\end{abstract}

Index Terms - Compressed Sensing, Quantization, Sampling, Uniform noise, Convex Optimization, Basis Pursuit.

\section{INTRODUCTION}

The theory of Compressed Sensing (CS) [1] enables reconstruction of sparse or compressible signals from a small number of linear measurements, relative to the dimension of the signal space. In this setting, knowledge of a signal $x \in \mathbb{R}^{N}$ is contained in the $m \leqslant N$ linear measurements provided by a sensing matrix $\Phi \in \mathbb{R}^{m \times N}$, i.e. we know only the $m$ inner products $\left\langle\varphi_{i}, x\right\rangle$; where $\left(\varphi_{i}\right)_{i=0}^{m-1}$ are the rows of $\Phi$.

In any realistic digital acquisition system, these analog measurements must be quantized before they may be stored or transmitted. The study of signal recovery from quantized measurements is thus of fundamental interest.

Therefore, in this paper we are interested in the noiseless and uniformly quantized sensing (or coding) model:

$$
y_{\mathrm{q}}=Q_{\alpha}[\Phi x]=\Phi x+n,
$$

L. J. is a Postdoctoral Researcher of the Belgian National Science Foundation (F.R.S.-FNRS). where $y_{\mathrm{q}} \in\left(\alpha \mathbb{N}+\frac{\alpha}{2}\right)^{m}$ is the quantized measurement vector, $\left(Q_{\alpha}[\cdot]\right)_{i}=\alpha\left\lfloor(\cdot)_{i} / \alpha\right\rfloor+\frac{\alpha}{2}$ is the uniform quantization operator in $\mathbb{R}^{m}$ of bin width $\alpha$, and $n \in \mathbb{R}^{m}$ is the quantization distortion. This model is a realistic description of systems where the quantization distortion dominates other secondary noise sources (e.g. thermal noise), an assumption valid for many electronic measurement devices.

A CS reconstruction program (or decoder) relies on the assumption that the sensed signal $x$ is sparse or compressible in an orthogonal ${ }^{1}$ basis $\Psi \in \mathbb{R}^{N \times N}$, i.e. the best $K$-term approximation $x_{K}$ in $\Psi$ is an exact or accurate representation of this signal even for small $K<N$. For simplicity, only the canonical basis $\Psi=\mathrm{Id}$ will be considered here.

The commonly used Basis Pursuit algorithm for CS recovery finds the sparsest signal (in $\ell_{1}$ norm) that could have produced the observed measurements. Directly using the quantized measurements in Basis Pursuit fails, however, as there may be no signal whose (unquantized) measurements reproduce the observed quantized values! This problem may be resolved by relaxing the data fidelity constraint. Using a quadratic constraint yields the standard Basis Pursuit DeNoise (BPDN) program [3]:

$$
\Delta\left(y_{\mathrm{q}}, \epsilon\right)=\underset{u \in \mathbb{R}^{N}}{\arg \min }\|u\|_{1} \text { s.t. }\left\|y_{\mathrm{q}}-\Phi u\right\|_{2} \leqslant \epsilon, \quad(\mathrm{BPDN})
$$

where $\|\cdot\|_{2}$ is the $\ell_{2}$-norm. The value of $\epsilon$ depends on the magnitude of quantization distortion, and should be chosen just large enough to ensure that the measurements of the original true signal satisfy the data fidelity constraint. In [3], an estimator of $\epsilon$ is obtained by considering $n$ as a uniform random vector $X$ with $X_{i} \sim_{\text {iid }} U\left(\left[-\frac{\alpha}{2}, \frac{\alpha}{2}\right]\right)$, i.e. $\epsilon^{2}=E\left[\|X\|_{2}^{2}\right]+$ $\kappa \sqrt{\operatorname{Var}\left[\|X\|_{2}^{2}\right]}=\frac{\alpha^{2}}{12} m+\kappa \frac{\alpha^{2}}{6 \sqrt{5}} m^{\frac{1}{2}}$. In that case, $u=x$ respects the constraint of BPDN with a probability higher than $1-e^{-c_{0} \kappa^{2}}$ for a certain constant $c_{0}>0$.

The stability of BPDN is guaranteed if the sensing matrix $\Phi \in \mathbb{R}^{m \times N}$ satisfies the Restricted Isometry Property (RIP) of order $K$ and radius $\delta \in(0,1)$, i.e. if there exists a constant $\mu$ such that $\mu \sqrt{1-\delta}\|u\|_{2} \leqslant\|\Phi u\|_{2} \leqslant \mu \sqrt{1+\delta}\|u\|_{2}$, for all $K$-sparse signals $u \in \mathbb{R}^{N}$. Generally, CS is described with

\footnotetext{
${ }^{1} \mathrm{~A}$ generalization for redundant basis, or dictionary, exists [2].
} 
normalized matrices $\bar{\Phi}=\Phi / \mu$ having unit-norm columns (in expectation) so that $\mu$ is absorbed in the normalizing constant.

Interestingly, Standard Gaussian Random (SGR) matrices, i.e. with entries drawn from $\Phi_{i j} \sim_{\text {iid }} N(0,1)$, satisfy the RIP with a controllable high probability (with $\mu=\sqrt{m}$ ), as soon as $m \geqslant O(K \log N / K)$ [3]. Moreover, other random constructions satisfying the RIP exist (e.g. Bernoulli matrix, Fourier ensemble, etc.) [1, 3].

For completeness, we include the following theorem expressing the aforementioned stability result, i.e. the $\ell_{2}-\ell_{1}$ instance optimality ${ }^{2}$ of BPDN.

Theorem 1 ([5]). Let $x \in \mathbb{R}^{N}$ be a compressible signal with a $K$-term $\ell_{1}$-approximation error $e_{0}(K)=K^{-\frac{1}{2}}\left\|x-x_{K}\right\|_{1}$, for $0 \leqslant K \leqslant N$, and $x_{K}$ the best $K$-term $\ell_{2}$-approximation of $x$. Let $\Phi$ be a RIP matrix of order $2 K$ and radius $0<$ $\delta_{2 K}<\sqrt{2}-1$. Given a measurement vector $y=\Phi x+n$ corrupted by a noise $n$ with power $\|n\|_{2} \leqslant \epsilon$, the solution $x^{*}=\Delta(y, \epsilon)$ obeys the $\ell_{2}-\ell_{1}$ instance optimality

$$
\left\|x^{*}-x\right\|_{2} \leqslant A e_{0}(K)+B \frac{\epsilon}{\mu},
$$

for values $A=2 \frac{1+(\sqrt{2}-1) \delta_{2 K}}{1-(\sqrt{2}+1) \delta_{2 K}}$ and $B=\frac{4 \sqrt{1+\delta_{2 K}}}{1-(\sqrt{2}+1) \delta_{2 K}}$. For instance, for $\delta_{2 K}=0.2, A<4.2$ and $B<8.5$.

However, using the BPDN decoder to account for quantization distortion is theoretically unsatisfying for several reasons. First, there is no guarantee that the BPDN solution $x^{*}$ respects Quantization Consistency (QC), i.e. $Q_{\alpha}\left[\Phi x^{*}\right]=y_{\mathrm{q}}$. This will be met iff $\left\|y_{\mathrm{q}}-\Phi x^{*}\right\|_{\infty} \leqslant \frac{\alpha}{2}$, which is not necessarily implied by the BPDN $\ell_{2}$ fidelity constraint. Second, from a Bayesian Maximum a Posteriori (MAP) standpoint, BPDN can be viewed as solving an ill-posed inverse problem where the $\ell_{2}$-norm used in the fidelity term corresponds to the conditional log-likelihood associated to an additive white Gaussian noise. However, the quantization distortion is not Gaussian, but rather uniformly distributed. This motivates the need for a new kind of CS decoder that more faithfully models the quantization distortion.

Recently, a few works have focused on this problem. In [6], the extreme case of 1-bit CS is studied, i.e. when only the signs of the measurements are sent to the decoder. Authors tackle the reconstruction problem by adding a sign consistency constraint in a modified BPDN program working on the sphere of unit-norm signals. In [7], an adaptation of both BPDN and the Subspace Pursuit integrates the QC constraint explicitly. However, despite interesting experimental results, no theoretical guarantees are given about the approximation error reached by these solutions. In oversampled ADC conversion of signal [8] and in image restoration problems [9], dequantization obtained from global optimization with equivalent QC constraint expressed in $\ell_{\infty}$-norm can also be found.

This paper, linked to the companion technical report [11], is structured as follows. In Section 2, we present a new class

\footnotetext{
${ }^{2}$ Adopting the definition of mixed-norm instance optimality [4].
}

of abstract decoders, coined Basis Pursuit DeQuantizer of moment $p\left(\mathrm{BPDQ}_{p}\right)$, that model the quantization distortion more faithfully. These decoders consist in minimizing the sparsity of the signal to be reconstructed while imposing a data fidelity term of bounded $\ell_{p}$-norm, for $2 \leqslant p \leqslant \infty$. Section 3 introduces a modified Restricted Isometry Property $\left(\mathrm{RIP}_{p}\right)$ expressed in the $\ell_{p}$-norm. With this tool, we then prove then the stability of the $\mathrm{BPDQ}_{p}$ programs, i.e. their $\ell_{2}-\ell_{1}$ instance optimality. In Section 4 , we show that, given a sufficient number of measurements, the approximation error due to quantization scales inversely with $\sqrt{p+1}$. Finally, Section 5 reports numerical simulations on signal and image reconstruction problems.

\section{BASIS PURSUIT DEQUANTIZERS}

We introduce a new class of optimization programs (or decoders) that generalize the fidelity term of the BPDN program to noises that follow a centered Generalized Gaussian Distribution (GGD) of shape parameter $p \geqslant 1$ [10], with the uniform noise case corresponding to $p \rightarrow \infty$. These decoders reconstruct an approximation of the sparse or compressible signal $x$ from its distorted measurements $y=\Phi x+n$ when the distortion has a bounded $p^{\text {th }}$ moment, i.e. $\|n\|_{p} \leqslant \epsilon$. Formally, the decoder writes

$$
\Delta_{p}(y, \epsilon)=\underset{u \in \mathbb{R}^{N}}{\arg \min }\|u\|_{1} \text { s.t. }\|y-\Phi u\|_{p} \leqslant \epsilon .
$$

We dub this class of decoders Basis Pursuit DeQuantizer of moment $p$ (or $\mathrm{BPDQ}_{p}$ ) since, as shown in Section 4, their approximation error when $\Phi x$ is uniformly quantized decreases as both the moment $p$ and the oversampling factor $m / K$ increase.

\section{RIP ${ }_{p}$ AND $\ell_{2}-\ell_{1}$ INSTANCE OPTIMALITY}

In order to study the approximation error of $\mathrm{BPDQ}_{p}$, we introduce the Restricted Isometry Property of moment $p$ (or $\mathrm{RIP}_{p}$ ).

Definition 1. A matrix $\Phi \in \mathbb{R}^{m \times N}$ satisfies the $R I P_{p}(1 \leqslant$ $p \leqslant \infty)$ property of order $K$ and radius $\delta$, if there exists a constant $\mu_{p}>0$ such that

$$
\mu_{p} \sqrt{1-\delta}\|x\|_{2} \leqslant\|\Phi x\|_{p} \leqslant \mu_{p} \sqrt{1+\delta}\|x\|_{2},
$$

for all $x \in \mathbb{R}^{N}$ with $\|x\|_{0} \leqslant K$, and where $\|\cdot\|_{p}$ is the $\ell_{p}$-norm on $\mathbb{R}^{m}$.

The common RIP previously introduced is thus the $\mathrm{RIP}_{2}$. Interestingly, SGR matrices $\Phi \in \mathbb{R}^{m \times N}$ satisfy also the $\mathrm{RIP}_{p}$ of order $K$ and radius $0<\delta<1$ with high probability provided that $m \geqslant O\left(\left(\delta^{-2} K \log N / K\right)^{p / 2}\right)$ for $2 \leqslant p<\infty$, or $\log m \geqslant O\left(\delta^{-2} K \log N / K\right)$ for $p=\infty$; see [11] for details. Moreover, for these matrices an asymptotic (in $m$ ) approximation for $\mu_{p}$ is

$$
\sqrt{2} \pi^{-\frac{1}{2 p}} \Gamma\left[\frac{p+1}{2}\right]^{\frac{1}{p}} m^{\frac{1}{p}}
$$




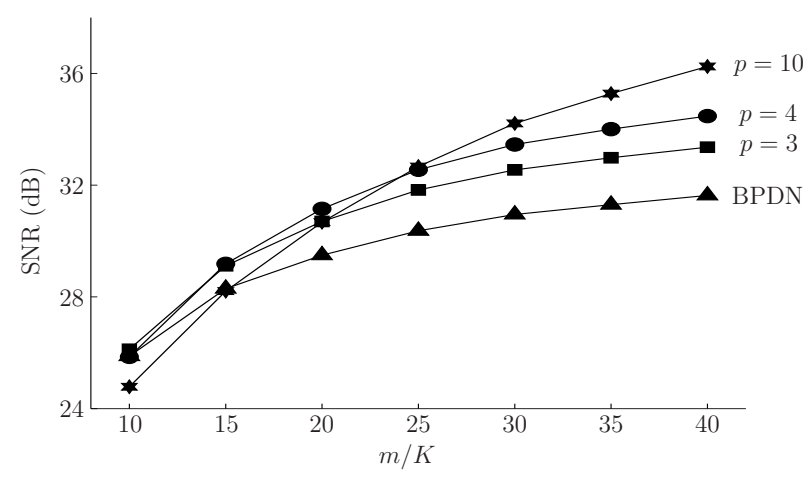

Fig. 1: Quality of $\mathrm{BPDQ}_{p}$ for different $m / K$ and $p$.

if $2 \leqslant p<\infty$ and $\mu_{\infty} \geqslant \rho^{-1} \sqrt{\log m}$ for a certain $\rho>0$ [11]. This results from the specialization to SGR vectors of a study made in [12]. We are now ready to state our main result.

Theorem 2. Let $x \in \mathbb{R}^{N}$ be a compressible signal with a $K$ term $\ell_{1}$-approximation error $e_{0}(K)=K^{-\frac{1}{2}}\left\|x-x_{K}\right\|_{1}$, for $0 \leqslant K \leqslant N$, and $x_{K}$ the best $K$-term $\ell_{2}$-approximation of $x$. Let $\Phi$ be a RIP matrix on s sparse signals with radius $\delta_{s}$, for $s \in\{K, 2 K, 3 K\}$ and $2 \leqslant p<\infty$. Given a measurement vector $y=\Phi x+n$ with $\|n\|_{p} \leqslant \epsilon$, the solution $x_{p}^{*}=\Delta_{p}(y, \epsilon)$ obeys the $\ell_{2}-\ell_{1}$ instance optimality

$$
\left\|x_{p}^{*}-x\right\|_{2} \leqslant A_{p} e_{0}(K)+B_{p} \frac{\epsilon}{\mu_{p}},
$$

for $A_{p}=\frac{2\left(1+C_{p}-\delta_{2 K}\right)}{1-\delta_{2 K}-C_{p}}, B_{p}=\frac{4 \sqrt{1+\delta_{2 K}}}{1-\delta_{2 K}-C_{p}}$, and with $C_{p}=$ $C_{p}\left(\delta_{K}, \delta_{2 K}, \delta_{3 K}\right)$ well behaved.

The approximation error reached by BPDQ is thus bounded in (5) by the sum of the compressibility and the noise errors. As shown in [11], this theorem uses explicitly the 2 -smoothness of the Banach spaces $\ell_{p}$ when $2 \leqslant p<\infty$ [13] and their possible embedding in $\ell_{2}$. The value $C_{p}$ behaves as $\sqrt{\left(\delta_{K}+\delta_{3 K}\right)\left(1+\delta_{2 K}\right) p}$ for large $p$, and as $\delta_{3 K}+\frac{3}{4}\left(1+\delta_{3 K}\right)(p-2)$ for $p \simeq 2$. For $p=2$, Theorem 2 reduces to Theorem 1 if $\delta_{3 K}=\sqrt{2} \delta_{2 K}$.

\section{QUANTIZATION ERROR REDUCTION}

We now turn to the behavior of the $\mathrm{BPDN}_{p}$ decoders on quantized measurements of sparse or compressible signals. First, assuming the quantization distortion $n=Q_{\alpha}[\Phi x]-\Phi x$ is uniformly distributed in each quantization bin, we proved in [11] that for

$$
\epsilon=\epsilon_{p}(\alpha) \triangleq \frac{\alpha}{2(p+1)^{1 / p}}(m+\kappa(p+1) \sqrt{m})^{\frac{1}{p}},
$$

$u=x$ is a solution of the $\mathrm{BPDQ}_{p}$ fidelity constraint with a probability at least $1-e^{-2 \kappa^{2}}$.

Second, by Theorem 2 , when $\Phi$ is $\operatorname{RIP}_{p}$ with $2 \leqslant p<\infty$, i.e. when for $m \geqslant O\left(\left(\delta^{-2} K \log N / K\right)^{p / 2}\right)$ for SGR matrices, we have

$$
\left\|x-x_{p}^{*}\right\|_{2} \leqslant A_{p} e_{0}(K)+B_{p} \frac{\epsilon_{p}(\alpha)}{\mu_{p}} .
$$

Third, from (4), we have the approximation $\mu_{p} \simeq c_{p} m^{\frac{1}{p}}$ with $c_{p}=\sqrt{2} \pi^{-\frac{1}{2 p}} \Gamma\left[\frac{p+1}{2}\right]^{\frac{1}{p}} \geqslant 2^{-\frac{1}{2}} e^{-\frac{3}{4}} \sqrt{p+1}(1+$ $\left.O\left(p^{-2}\right)\right)$, using Stirling formula $\Gamma(z)=\left(\frac{2 \pi}{z}\right)^{\frac{1}{2}}\left(\frac{z}{e}\right)^{z}(1+$ $\left.O\left(\frac{1}{z}\right)\right)$.

Finally, bounding the different functions involved yields

$$
\frac{\epsilon_{p}(\alpha)}{\mu_{p}} \lesssim C \frac{\alpha}{\sqrt{p+1}}\left(1+O\left(p^{-2}\right)\right), \quad C<1.497
$$

In short, the noise error term in the $\ell_{2}-\ell_{1}$ instance optimality relation (7) for the quantized model (1) is thus divided by $\sqrt{p+1}$ if the sensing matrix $\Phi$ satisfies the RIP ${ }_{p}$ !

More precisely, with a philosophy close to the oversampled ADC conversion [8], this error noise reduction happens in oversampled sensing, i.e. when the oversampling factor $m / K$ is high. Indeed, in that case a SGR matrix $\Phi$ satisfies the $\mathrm{RIP}_{p}$ with high probability for high $p$. Moreover, oversampling gives a smaller $\delta$, i.e. $\delta \propto m^{-1 / p}$, hence counteracting the increase of $p$ in the factor $C_{p}$ of the values $A_{p} \geqslant 2$ and $B_{p} \geqslant 4$. This decrease of $\delta$ also favors BPDN, but since the value $A=A_{2}$ and $B=B_{2}$ in (2) are bounded from below this effect is limited. This is confirmed experimentally in Section 5.

Finally, note that the necessity of satisfying $\mathrm{RIP}_{p}$ implies that we cannot directly set $p=\infty$ in $\mathrm{BPDQ}_{p}$ to impose Quantization Consistency (QC) of this decoder ${ }^{3}$. Indeed, for a given oversampling factor $m / K$, a SGR matrix $\Phi$ can be $\operatorname{RIP}_{p}$ only over a finite interval $p \in\left[2, p_{\text {max }}\right]$.

\section{EXPERIMENTAL RESULTS}

The $\mathrm{BPDQ}_{p}$ decoders are practically solved by monotone operator splitting proximal methods $[14,11]$. More precisely, as both the $\ell_{1}$-norm and the indicator function of the constraint in $\mathrm{BPDQ}_{p}$ are non-differentiable, the DouglasRachford splitting is used. The Douglas-Rachford recursion to solve $\mathrm{BPDQ}_{p}$ can be written in the compact form

$u^{(t+1)}=\left(1-\frac{\alpha_{t}}{2}\right) u^{(t)}+\frac{\alpha_{t}}{2}\left(2 S_{\gamma}-\operatorname{Id}\right) \circ\left(2 \mathcal{P}_{T_{p}(\epsilon)}-\mathrm{Id}\right)\left(u^{(t)}\right)$, where $\alpha_{t} \in(0,2), \forall t \in \mathbb{N}, \gamma>0, S_{\gamma}$ is the componentwise soft-thresholding operator with threshold $\gamma$ and $\mathcal{P}_{T_{p}(\epsilon)}$ is the orthogonal projection onto the closed convex constraint set $T_{p}(\epsilon)=\left\{u \in \mathbb{R}^{N}:\left\|y_{\mathrm{q}}-\Phi u\right\|_{p} \leqslant \epsilon\right\}$. From [14], one can show that the sequence $\left(u^{(t)}\right)_{t \in \mathbb{N}}$ converges to some point $u^{*}$ and $\mathcal{P}_{T_{p}(\epsilon)}\left(u^{*}\right)$ is a solution of $\mathrm{BPDQ}_{p}$. The projection $\mathcal{P}_{T_{p}(\epsilon)}$ was computed iteratively using Newton's method to solve the Lagrange multiplier equations arising from minimizing the distance to the constraint set.

For the first experiment, setting the dimension $N=1024$ and the sparsity level $K=16$, we have generated $500 \mathrm{~K}$ sparse signals with support selected uniformly at random in $\{1, \cdots, N\}$. The non-zero elements have been drawn from a standard Gaussian distribution $N(0,1)$. For each sparse signal, $m$ quantized measurements have been recorded as in

\footnotetext{
${ }^{3}$ Observing also that $\lim _{p \rightarrow \infty} \epsilon_{p}(\alpha)=\alpha / 2$.
} 


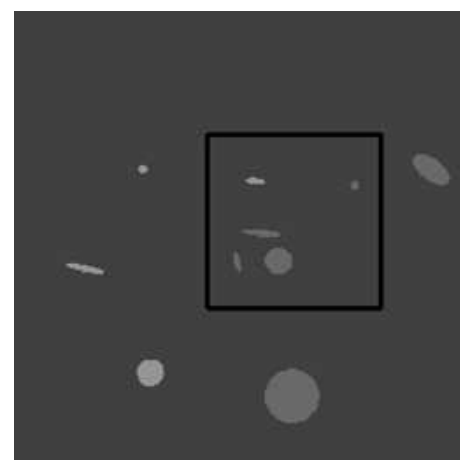

(a)

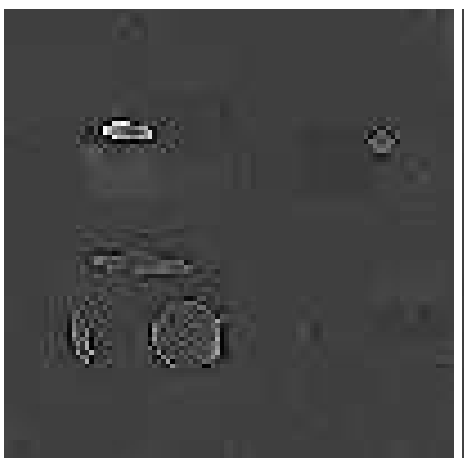

(b) $(\mathrm{SNR}=2.96 \mathrm{~dB})$

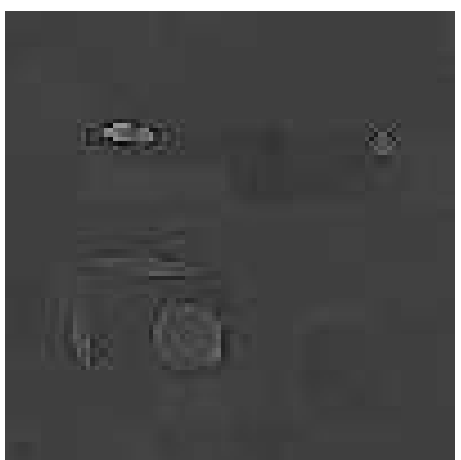

(c) $(\mathrm{SNR}=3.93 \mathrm{~dB})$

Fig. 2: Reconstruction from quantized undersampled Fourier measurements. (a) Original; details from (b) BPDN; and (c) BPDQ 10

model (1) with a SGR matrix $\Phi \in \mathbb{R}^{m \times N}$. The bin width has been set to $\alpha=\|\Phi x\|_{\infty} / 40$. In Figure 1, we plot the average quality of the reconstructions of $\mathrm{BPDQ}_{p}$ for various $p \geqslant 2$ and $m / K \in[10,40]$. We use the quality metric $\operatorname{SNR}(\hat{x} ; x)=20 \log _{10} \frac{\|x\|_{2}}{\|x-\hat{x}\|_{2}}$, where $x$ is the true original signal and $\hat{x}$ the reconstruction. The different decoders become dominant from oversampling factors $m / K$ increasing with $p$. This confirms the fact that the noise error can be reduced when both $p$ and $m / K$ are high.

In the second experiment, we applied our methods to a model undersampled MRI reconstruction problem. Using an example similar to [15], the original signal is a 256 by 256 pixel "simulated angiogram" comprised of 10 randomly placed ellipses. The linear measurements are the real and imaginary components of one sixth of the Fourier coefficients at randomly selected locations in Fourier space, giving $m=256^{2} / 6$ independent measurements. These are quantized with a bin width $\alpha$ giving at most 12 quantization levels for each measurement. We use the Haar wavelet transform as a sparsity basis. The measurement matrix is then $\Phi=F \Psi$, where $\Psi$ is the Haar matrix, and $F$ is formed by the randomly selected rows of the Discrete Fourier Transform matrix. The original image has $K=821$ nonzero wavelet coefficients, giving an oversampling ratio $m / K=13.3$. In Figure 2, we show 100 by 100 pixel details of the results of reconstruction with BPDN, and with BPDQ for $p=10$. Note that we do not have any proof that the sensing matrix $\Phi$ satisfies the $\operatorname{RIP}_{p}$ (3). We nonetheless obtain similar results as in the previous 1-d example. The BPDQ reconstruction shows improvements both in SNR and visual quality compared to BPDN.

\section{CONCLUSION}

The objective of this paper was to show that the BPDN reconstruction program commonly used in Compressed Sensing with noisy measurements is not always adapted to quantization distortion. We introduced a new class of decoders, the Basis Pursuit DeQuantizers, and we have shown both theoretically and experimentally that $\mathrm{BPDQ}_{p}$ exhibit an substantial reduction of the approximation error in oversampled situations. An interesting perspective is to characterize the evolution of the optimal moment $p$ with the oversampling ratio. This could allow the selection of the best BPDQ decoder as a function of the precise CS coding/decoding scenario.

\section{REFERENCES}

[1] E.J. Candès and J. Romberg, "Quantitative Robust Uncertainty Principles and Optimally Sparse Decompositions," Found. Comp. Math., 6(2):227-254, 2006.

[2] H. Rauhut, K. Schnass, and P. Vandergheynst, "Compressed sensing and redundant dictionaries," IEEE T. Inform. Theory., 54(5):22102219, 2007.

[3] E. Candès, J. Romberg, and T. Tao, "Stable signal recovery from incomplete and inaccurate measurements," Comm. Pure Appl. Math, 59(8):1207-1223, 2006.

[4] A. Cohen, R. DeVore, and W. Dahmen, "Compressed sensing and best k-term approximation," J. Amer. Math. Soc., 22:211-231, 2009.

[5] E. Candès, "The restricted isometry property and its implications for compressed sensing," Compte Rendus de l'Academie des Sciences, Paris, Serie I, 346:589-592, 2008.

[6] P. Boufounos and R.G. Baraniuk, "1-bit compressive sensing," in 42nd Conf. Inf. Sc. Systems (CISS), Princeton, NJ, March 2008, 19-21.

[7] W. Dai, H. Vinh Pham, and O. Milenkovic, "Distortion-rate functions for quantized compressive sensing," preprint, 2009, arXiv:0901.0749.

[8] N.T. Thao and M. Vetterli, "Deterministic analysis of oversampled A/D conversion and decoding improvement based on consistent estimates," IEEE. T. Sig. Proc., 42(3):519-531, 1994.

[9] P. Weiss, L. Blanc-Feraud, T. Andre, and M. Antonini "Compression artifacts reduction using variational methods: Algorithms and experimental study Acoustics," IEEE ICASSP 2008, 1173-1176.

[10] M.K. Varanasi and B. Aazhang, "Parametric generalized Gaussian density estimation," Journ. Acoust. Soc. Amer, 86:1404-1415, 1989.

[11] L. Jacques, D.K. Hammond, and M.J. Fadili, "Dequantizing compressed sensing," Tech. Rep., 2009, http://www.tele.ucl. ac.be/ jacques/preprint/TR-LJ-2009.01.pdf.

[12] D. François, V. Wertz, and M. Verleysen, "The Concentration of Fractional Distances," IEEE T. Know. Data. Eng., 873-886, 2007.

[13] W.L. Bynum, "Weak parallelogram laws for Banach spaces," Canad. Math. Bull., 19(3):269-275, 1976.

[14] P.L. Combettes, "Solving monotone inclusions via compositions of nonexpansive averaged operators," Optimization, 53:475-504, 2004.

[15] M. Lustig, D. Donoho and J. M. Pauly, "Sparse MRI: The Application of Compressed Sensing for Rapid MR Imaging," Magnetic Resonance in Medicine, 58:1182-1195, 2007. 